\title{
REVIEW
}

\section{Targeting RANKL in metastasis}

\section{William C Dougall ${ }^{1}$, Ingunn Holen ${ }^{2}$ and Eva González Suárez ${ }^{3}$}

\author{
${ }^{1}$ Therapeutic Innovation Unit, Amgen Inc, Seattle, WA, USA. ${ }^{2}$ Academic Unit of Clinical Oncology, Medical School, \\ University of Sheffield, Sheffield, England. ${ }^{3}$ Cancer Epigenetics and Biology Program PEBC, Bellvitge Institute for \\ Biomedical Research, IDIBELL, Barcelona, Spain.
}

\begin{abstract}
Acting through its cognate receptor, receptor activator of nuclear factor- $\kappa B$ (RANK), RANK ligand (RANKL) is an essential mediator of osteoclast function and survival. Preclinical data have now firmly established that blockade of tumorinduced osteoclastogenesis by RANKL inhibition will not only protect against bone destruction but will also inhibit the progression of established bone metastases and delay the formation of de novo bone metastases in cancer models. In patients with bone metastases, skeletal complications are driven by increased osteoclastic activity and may result in pathological fractures, spinal cord compression and the need for radiotherapy to the bone or orthopedic surgery (collectively known as skeletal-related events (SREs)). Denosumab, a fully human monoclonal antibody against RANKL, has been demonstrated to prevent or delay SREs in patients with solid tumors that have metastasized to bone. In addition to its central role in tumor-induced osteolysis, bone destruction and skeletal tumor progression, there is emerging evidence for direct pro-metastatic effects of RANKL, independent of osteoclasts. For example, RANKL also stimulates metastasis via activity on RANK-expressing cancer cells, resulting in increased invasion and migration. Pharmacological inhibition of RANKL may also reduce bone and lung metastasis through blockade of the direct action of RANKL on metastatic cells. This review describes these distinct but potentially overlapping mechanisms by which RANKL may promote metastases.
\end{abstract}

BoneKEy Reports 3, Article number: 519 (2014) | doi:10.1038/bonekey.2014.14

\section{Introduction}

Receptor activator of nuclear factor (NF)-kB ligand (RANKL) and its cognate receptor RANK were initially characterized as essential factors for osteoclastogenesis (reviewed in Lacey et al. ${ }^{1}$ ). Mice in which either the RANK or RANKL genes are ablated exhibit severe osteopetrosis and an absence of osteoclasts. ${ }^{2,3}$ RANKL and myeloid colony-stimulating factor are sufficient to generate osteoclasts from their myeloid precursors. ${ }^{4}$ Osteoprotegerin (OPG), a decoy receptor for RANKL, inhibits osteoclasts and acts as a physiological regulator of bone resorption by counterbalancing RANKL activity. ${ }^{5}$ The discovery of RANKL, RANK and OPG revealed the molecular mechanisms critical for osteoclastogenesis and the physiological control of bone remodeling.

Skeletal metastasis results from reciprocal engagement between tumor cells and normal host cells of the bone microenvironment (for example, osteoclasts, stromal cells, vascular cells and so on; Figure 1). The resultant bone destruction and skeletal complications are mediated by a pathologically increased rate of bone remodeling driven by marked increases in osteoclast activity. This cooperative interaction between the bone microenvironment and tumor is known as the vicious cycle and is critical for metastatic establishment and progression. ${ }^{6}$ Thus, the increased bone turnover driven by osteoclastic bone resorption not only contributes to skeletal morbidity, but has also been hypothesized to contribute to both early tumor colonization and later progression in bone. Data supporting this hypothesis have been comprehensively described in a recent review by Weilbaecher et al. ${ }^{7}$ Given that RANKL is essential for osteoclastogenesis, it is also an attractive therapeutic target for the prevention and treatment of metastatic bone disease.

The generation of the RANK- and RANKL-knockout mice revealed that, other than osteopetrosis and peripheral lymph node agenesis, the only tissue compartment that exhibited obvious functional defects was the development of the mammary gland during pregnancy. ${ }^{8}$ In the absence of RANK or $R A N K L$, lactation is impaired owing to a defective expansion of the lobulo-alveolar mammary epithelium at midgestation. This defect is associated with reduced mammary epithelial proliferation and survival, and it is now understood that RANKL is the major paracrine effector of progesterone's mitogenic action in mouse mammary and human breast epithelium. ${ }^{9,10}$ RANKL protein is regulated by progesterone and is expressed within the

Correspondence: Dr WC Dougall, Therapeutic Innovation Unit, Amgen, Inc, 1201 Amgen Ct. West, Seattle, WA 98119, USA.

E-mail: dougallw@amgen.com 


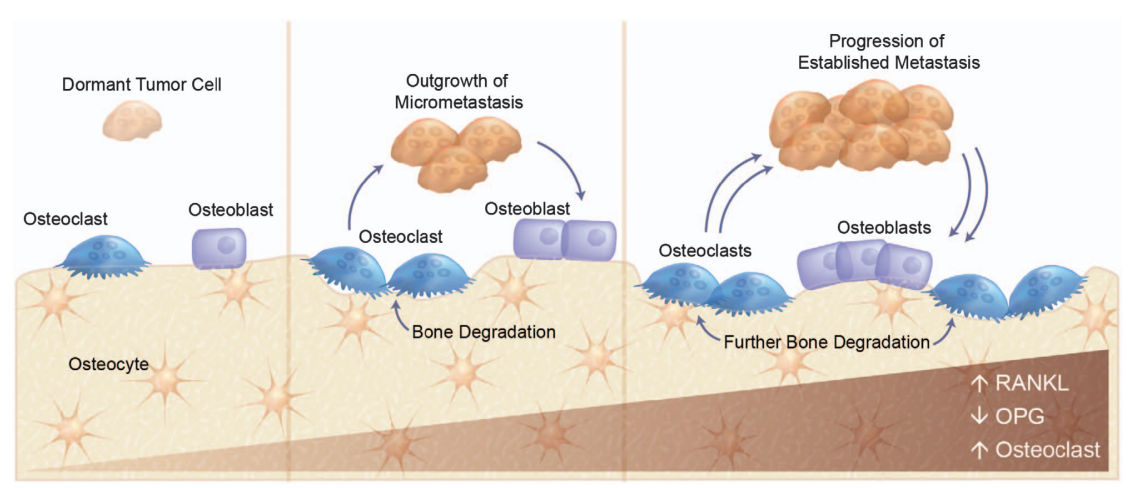

Figure 1 Interaction with the bone microenvironment supports tumor metastasis. OPG, osteoprotegerin; RANKL, RANK ligand.

estrogen receptor-positive/progesterone receptor-positive $(\mathrm{ER}+/ \mathrm{PR}+)$ luminal epithelium. ${ }^{10,11}$ RANKL is positively correlated with breast proliferation and acts in a paracrine manner on RANK expressed in proliferating ER - /PR - breast cells. ${ }^{9,11}$ In addition to mediating the mitogenic effect of progesterone, RANKL also controls the responsiveness of mammary stem cell and luminal progenitors ${ }^{12}$ to progesterone. ${ }^{13,14}$ These observations provided the initial rationale that RANKL might also directly stimulate tumor formation and metastatic progression through activation of epithelial cells.

Here we will review the preclinical in vivo and in vitro mechanistic evidence that RANKL blockade effectively inhibits both skeletal and nonskeletal metastasis. We will also review the clinical trials that have addressed the potential for RANKL inhibition to delay or prevent skeletal complications or bone metastases in cancer patients.

\section{Role of RANKL in Pathologically-Induced Osteolysis and Bone Metastasis}

In bone metastasis, RANKL-positive stromal cells have been observed at the tumor/bone interface adjacent to osteoclasts $^{15,16}$ and associated with tumor-induced osteolysis. ${ }^{17}$ Early work using co-culture approaches demonstrated that, in contrast to the observations in progesterone-exposed mammary/breast tissue and primary breast cancer specimens (see below), breast tumor cells cultured in vitro did not express RANKL but could provoke increased stromal RANKL expression, leading to enhanced osteoclast formation. ${ }^{18}$ This paradigm of stromal reaction to tumor involvement has been consistently observed across different model systems. The molecular mechanisms for upregulation of RANKL within bone stroma include transcriptional responses to tumor-associated cytokines and factors including interleukin (IL)-1 $\beta, \mathrm{IL}-6, \mathrm{IL}-8$, IL-11, IL-17, macrophage inflammatory protein-1 $\alpha$, tumor necrosis factor- $\alpha$, parathyroid hormone-related protein (PTHrP), prostaglandin E2 (PGE2) and CXCL13. ${ }^{19,20}$ Interestingly, stimulation of the sympathetic nervous system has also been demonstrated to increase RANKL levels within osteoblast lineage cells. ${ }^{12}$ Factors produced by tumors such as PTHrP, IL-1, PGE2, DKK-1 or epidermal growth factor also cause a decrease in local OPG expression by stromal and osteoblastic cells, thereby reducing local levels of the natural RANKL inhibitor. ${ }^{18,21,22}$ Altogether, the net impact of tumor involvement on bone is to increase the RANKL:OPG ratio.
Reactive changes in the bone microenvironment to tumor involvement leads to locally increased RANKL:OPG levels, and experimental observations in bone metastasis indicate that this is the predominant RANKL signal leading to focally aggressive osteolytic bone destruction. The relative contributions of tumor cell-expressed RANKL and/or OPG to the net RANKL:OPG ratio in bone metastasis is less well defined. Tumor cells themselves can express OPG, and this can be modulated in the bone by estrogen signaling. ${ }^{23,24}$ Although the preponderance of data indicates little (or variable) RANKL expression on tumor cells themselves, RANKL expression has been occasionally reported in primary tumors or tumor cell lines (reviewed in Dougall ${ }^{19}$ ), which would be predicted to cause osteolysis directly, leading to greater bone metastasis. Lack of specificity in reagents used to detect RANKL could explain the disparate observations. Expression analysis of RANKL in human primary tumors and potential relationships to metastatic outcomes are discussed later in this review but it is important to consider that the degree of RANKL expression observed in the primary tumor may not reflect the local influences on RANKL levels at the bone metastatic site. Bone microenvironment signals could induce RANKL or suppress OPG expression by metastatic tumor cells using mechanisms similar to those observed in stromal cells. Experimental methods using either real-time imaging of RANKL promoter/reporter constructs or careful analysis of host/tumor mRNA have shown that the bone environment can induce RANKL within prostate tumor, ${ }^{25}$ head and neck squamous cell carcinoma (HNSCC) ${ }^{26}$ or oral squamous carcinoma ${ }^{27}$ cells (OSCC). In addition to cytokines/signals known to stimulate RANKL production or suppress OPG expression in stroma, other stimuli present in bone-such as $\alpha \mathrm{v} \beta 3$ integrin, CD44, TGF- $\beta$-dependent signaling ${ }^{28}$ or the hypoxic environment of bone metastasis, ${ }^{29}$-may operate on metastatic tumor cells, leading to enhanced RANKL production. Interestingly, it has been hypothesized that the reactive skeletal environment may activate micrometastases to progress into overt bone metastasis by provoking epithelial-to-mesenchymal transition (EMT) of tumor cells. ${ }^{30}$ The expression of RANKL on tumor cells undergoing EMT, as has been observed in prostate cancer ${ }^{31}$ and HNSCC, ${ }^{26}$ may be part of the EMT activation of metastatic outgrowth by further exacerbating bone resorption.

The in vitro observations that RANKL expressed on tumor cells can mediate osteoclastogensis directly without any requirement for osteoblasts or stromal cells (for example, Zhang et al. ${ }^{32}$ ) suggest that tumor-expressed RANKL, perhaps locally 
induced within the skeleton, would contribute to bone metastasis beyond the stromal contribution. However, experimental evidence demonstrates that any tumor-expressed RANKL has relatively minimal (or variable) contributions (above and beyond stromal RANKL) to bone metastasis in vivo. For instance, by comparing a human-specific RANKL antibody with OPG-Fc, which inhibits both human and mouse RANKL, Morrissey et al., ${ }^{33}$ demonstrated that the stromal RANKL mediates bone destruction in a human prostate cancer xenograft model in mice. Using a similar approach in models of OSCC, Sato et al. ${ }^{34}$ demonstrated that the stromal contribution of RANKL was consistently observed, whereas involvement of tumor cell RANKL in osteolysis was variable. These observations suggest the potential for tumor-expressed RANKL to further amplify the osteoclastic response in bone metastasis or to provoke bone resorption at early steps in bone colonization.

Pharmacological RANKL inhibitors would be active against RANKL, irrespective of the source (for example, bone stroma, infiltrating cells and tumor cells), and preclinical experimental bone metastasis studies using OPG-Fc or RANK-Fc support an essential role for RANKL in tumor-associated osteoclastogenesis and subsequent osteolytic bone destruction. Tumorassociated bone destruction in bone metastasis models of breast, lung, prostate, renal, OSCC, colon and epidermoid tumors is prevented by pharmacological inhibition of RANKL (reviewed in Roodman and Dougall ${ }^{35}$ ). Most studies have examined the role of RANKL in bone destruction and progression of established bone metastases; however, the reciprocal feedback of tumor cells and bone resorption might also actively contribute to tumor colonization and metastatic outgrowth, suggesting a role for RANKL at earlier stages of bone metastasis. Recent evidence from an in vivo model system has demonstrated that the localization of MDA-MB-231 breast cancer cells into bone induces significant contact-dependent changes of both osteoclast and osteoblast numbers before the development of bone lesions. ${ }^{36}$ Defining the molecular mechanisms responsible for these early events is challenging, partly because of the limitations in technological identification of small numbers of tumor cells within bone. Only detailed histological analysis will reveal the cellular composition of small tumor foci, and subsequent identification of specific molecules associated with different cell types within these foci requires well-validated antibodies. Experimental models have been utilized to address whether skeletal metastases might be prevented or delayed through pharmacological blockade of RANKL and bone turnover in which OPG-Fc treatment was initiated either simultaneously with or before tumor cell-line inoculation. RANKL inhibition significantly delayed de novo formation of skeletal metastases. ${ }^{37}$ In total, these data indicate that diverse cancer types employ multiple mechanisms involving RANKL as a common signaling pathway for tumorinduced osteolysis. Moreover, RANKL actively contributes to the vicious cycle of bone metastasis, mediating the processes of early tumor colonization and progression of bone metastasis.

\section{Direct (Osteoclast-Independent) Pro-Metastatic Effects of RANKL}

The observation that mouse mammary gland development during pregnancy is controlled by the intrinsic expression and functionality of RANK and RANKL within mammary tissue revealed a critical role for the RANKL pathway in epithelial biology. These findings provided the first experimental rationale that RANKL might directly control carcinogenesis and metastatic progression, distinct from its role in the promotion of osteoclastogenesis (Figure 2). In fact, the critical direct contribution of RANKL to breast tumor initiation and distant metastasis has now been demonstrated in vivo. During tumor formation, RANKL promotes the increased proliferation and survival of the normal and preneoplastic breast as well as the proliferative and nonproliferative (regenerative) expansion of the mammary stem/progenitor cell. ${ }^{38-40}$ In combination, these RANKL-dependent mechanisms could expand the pool of breast epithelial cells undergoing transformation, thereby increasing mammary cancer risk. In support of this hypothesis, early RANKL inhibition profoundly blocks mammary tumor formation. ${ }^{38,39}$ In addition, both in vitro and in vivo studies have indicated that RANKL can also promote distant metastasis via direct effects on RANK-expressing cells (Figure 2). In this section, we will review the in vivo data supportive of RANKL promotion of distant metastasis, followed by a review of mechanistic data suggesting that RANKL promotes metastasis

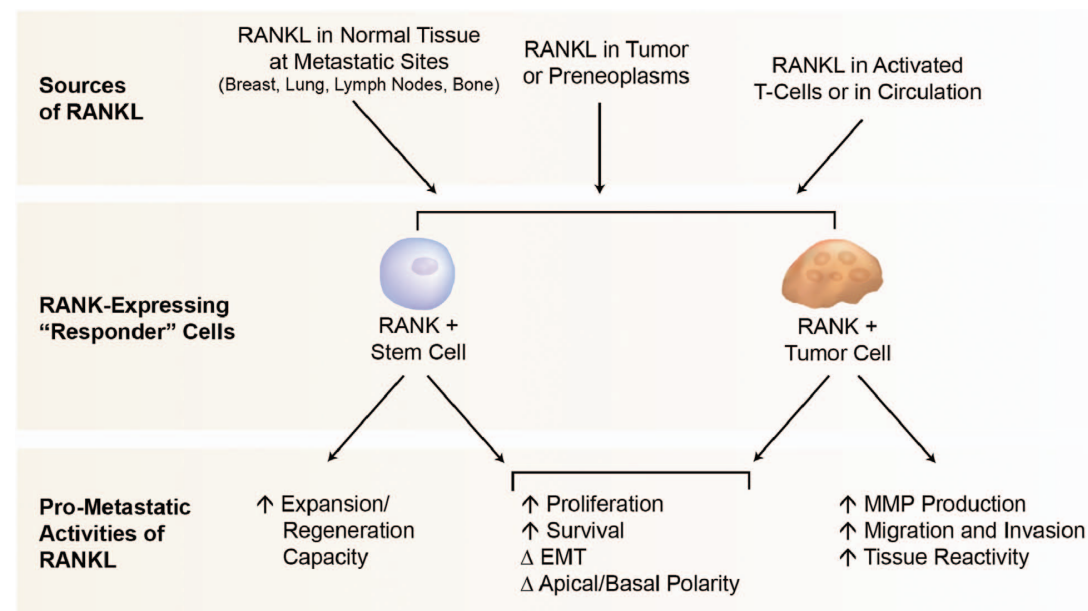

Figure 2 Mechanisms of RANKL-mediated promotion of metastasis. EMT, epithelial-to-mesenchymal transition; MMP, matrix metalloproteinase; RANKL, RANK ligand. 
by acting directly on RANK-expressing cells, including tumor cells or a stem/progenitor cell compartment.

Working from the observation that RANKL directly stimulates B16F10 melanoma cell migration in vitro (see below), Jones et al. ${ }^{41}$ tested whether a RANKL inhibitor would block B16F10 metastasis in vivo in an experimental bone metastasis model. Significant reductions in B16F10 skeletal metastasis were observed following OPG-Fc treatment, given simultaneously with tumor cell injection. Two lines of evidence from this study support the osteoclast-independent nature of this antimetastatic effect: first, the B16F10 subclone used did not stimulate a substantial osteoclast reaction to tumor cells in the bone; and second, in contrast to OPG-Fc, the bisphosphonate osteoclast inhibitor, zoledronic acid, did not reduce B16F10 bone metastasis. These observations are consistent with a direct role for RANKL in bone metastasis independent of its osteoclastogenic activity. This intracardiac experimental model predominately develops skeletal metastasis, which limits any inference on RANKL function toward metastasis to other sites. Moreover, it is difficult to experimentally dissect the distinct contributions of osteoclast-dependent and -independent effects of RANKL on metastasis within the bone compartment. ${ }^{42}$

Studies on RANKL function in lung metastasis models avoid the experimental challenges of discriminating between the indirect (for example, osteoclast-mediated) vs direct action (for example, acting on RANK-expressing cells) of RANKL function in the bone compartment. Data from these models provide strong evidence for direct promotion of metastasis, distinct from RANKL effects on osteoclasts. For instance, GonzalezSuarez et al. ${ }^{38}$ recently evaluated mouse mammary tumor virusneu (MMTV-neu) transgenic mice as a model to test the role of RANKL in spontaneous tumor development and lung metastases. In MMTV-neu mice, RANK-Fc treatment did not change median time to mammary tumor formation, but reduced the number of mammary tumors per mouse and significantly decreased spontaneous lung metastases. Tan et al. ${ }^{43}$ confirmed these findings using complementary methods, observing significantly fewer (50\%) spontaneous lung metastases in MMTV-neu mice crossed to the RANK-knockout mice vs MMTV-neu controls. ${ }^{43}$ Using an orthotopic mammary tumor cell line (MT2) that spontaneously metastasizes to lungs, Tan et al. ${ }^{43}$ also showed that treatment of mice with RANKL after MT2 tumor grafting to mammary gland increased the incidence and number of pulmonary metastases. Finally, knockdown of RANK within MT2 cells reduced lung metastases and inhibited the response to RANKL, suggesting that RANKL acted directly on RANK-expressing tumor cells.

'Gain-of-function' experiments in vivo have also provided evidence that the intrinsic expression of RANK on tumor cells enhances distant metastasis. For example, overexpression of RANK in MDA-MB-436 breast tumor cells increased the frequency and size of lung metastases after tail vein injection. ${ }^{44}$ Similarly, a significantly greater metastatic growth rate in the bone was observed in high RANK-expressing MDA-MB-231 breast cells compared with RANK-negative MDA-MB-231 cells. ${ }^{45}$ In both studies, the enhanced metastatic progression of RANK-expressing tumor cells at distant sites was much greater than tumor growth observed upon subcutaneous/orthotopic injection of tumor cells. In addition, knockdown of RANK expression in MDA-MB-231 cells prevented the increase in bone metastasis caused by activation of the sympathetic nervous system. ${ }^{12}$ These observations are consistent with the expression of RANKL at sites of lung and bone metastasis (and not within subcutaneous tumors) and suggest a direct prometastatic role for RANKL independent of tumor growth.

There is considerable in vitro and in vivo evidence for various pleiotropic mechanisms that would explain the direct (osteoclast-independent) stimulation of metastasis by RANKL. Multiple recent studies have demonstrated functional RANK protein expression on the surface of various cancer cell lines, and in vitro experiments suggest that RANKL stimulation will directly induce molecular pathways associated with tumor cell metastasis. Exposure of RANK-expressing human breast, lung, melanoma and prostate cancer cell lines to RANKL in vitro resulted in increased migration and invasion through collagen or fibronectin matrices. ${ }^{41,44,46-50}$ The RANKL-dependent effect is correlated with upregulation of several factors involved in migration, angiogenesis and invasion. For instance, RANKLdependent changes in MDA-231 breast cancer cells include matrix metalloproteinase-1(MMP1), ${ }^{46}$ MMP9, the MMP inducer EMMPRIN/CD47 and vascular endothelial growth factor (VEGF). ${ }^{51}$ The RANKL-stimulated migration and invasion of PC3 cells was correlated with increases in MMP9, IL-6, IL-8, VEGF and several members of the CXCL-family of chemokines, ${ }^{48}$ whereas migration and invasion of A549 lung cancer cells induced by RANKL was correlated with increases in ICAM-1 expression. ${ }^{50}$ Treatment of MT2 mammary tumor-bearing mice with RANKL resulted in decreased levels of maspin, a mammary serine protease inhibitor known to suppress metastases to diverse organs such as the lymph node, liver and lung. ${ }^{52}$ In addition, EMT is known to be associated with greater motility, invasion and metastasis, and data suggest that RANKLmediated EMT may also stimulate metastatic behavior. Constitutive activation of RANK induces EMT in the breast cell lines MCF10A and MDA-MB-436. The ability of RANK overexpression to increase pulmonary metastasis of MDA-MB-436 breast cancer cells was associated with EMTand increased cell migration and anchorage-independent growth. ${ }^{44}$

\section{Potential for Direct Pro-Metastatic Effect of RANKL via Action on Stem Cells}

In breast cancer, the recently established role of RANKL in the proliferation and regenerative potential of normal mammary stem cells or early breast epithelial progenitor cells ${ }^{13,14}$ suggests that RANKL-dependent activation of a cancer stem cell (CSC) or tumor-initiating cell (TIC) compartment may influence metastatic outcomes (Figure 2). The ability of RANKL to control canonical and noncanonical NF- $\kappa$ B pathways in mammary epithelia is also suggestive of a link to $\operatorname{CSC}^{53}$ as these latter pathways are functionally linked to breast CSC renewal. ${ }^{54}$ Although unproven, it is hypothesized that CSC, given their distinct features from the bulk of proliferating tumor cells, are responsible for metastatic disease and resistance to therapies. ${ }^{55}$ RANKL clearly regulates the activity of normal stem and descendant progenitor cells of the breast, ${ }^{13,14,40}$ but what is the evidence that this pathway influences breast CSC? After genetic inactivation of RANK within the mammary epithelial lineage, the self-renewal capacity of the Lin-CD24 + CD49fhi stem cell-enriched population isolated from mammary tumors was substantially reduced, correlating with the overall reduction 
in mammary tumor incidence observed in these mice after medroxyprogesterone acetate and 7,12-dimethylbenz $[\alpha]$ anthracene treatment. ${ }^{39}$ As spontaneous metastases do not occur in this particular model, the association of this stem cellenriched population with metastatic potential could not be determined. Using the experimental model of RANK overexpression in breast cells, Palafox et al. ${ }^{44}$ observed increased expression of breast CSC and basal/stem cell markers and functional effects consistent with enhanced stem cell activities. Overexpression of RANK in immortalized but nontumorigenic MCF-10 cells increased the frequency of CD44 + CD24 - cells and the fraction of cells expressing either CD133 or CD10, phenotypic markers consistent with a stem cell-like fraction. ${ }^{42}$ RANK overexpression in MCF10A cells confers the ability to grow in an anchorage-independent manner, a characteristic of tumor or stem cells. Stem cell functionality was also confirmed in RANK-overexpressing MCF-10 cells by demonstrating mammary outgrowth capacity after transplantation in vivo. In these experiments, RANK overexpression was also associated with increased tumorigenesis and metastasis suggesting that the observed increases in CSC numbers and activity may be relevant. HER-2 expression, a marker associated with breast CSC in humans ${ }^{56}$ or TIC in mice ${ }^{53}$ has now been shown to be modulated by the bone microenvironment. In a model mimicking the bone/tumor interactions, a co-culture of osteoblasts and MCF-7 breast cancer cells caused an increase in HER-2 that could be blocked by a RANKL inhibitor. RANKL treatment of MCF-7 cells increased the mammosphere-forming capacity, consistent with an effect on the stem cell activity.

\section{Analysis of Human RANKL Pathway Expression and Association with Metastasis}

An association of RANK/RANKL levels in tumors with patient outcome could support the pro-metastatic role of this pathway as described above. In primary breast cancer, RANK expression is more commonly observed in ER - /PR - tumors $(50 \%)$ than ER + /PR + tumors (18\%). ${ }^{44}$ Higher RANK levels are positively associated with both higher proliferation index and pathological grade in primary breast cancer. ${ }^{44}$ RANK/RANKL mRNA expression levels (determined by reverse transcription (RT)-PCR) were able to discriminate between node-negative and lymph node-positive/distant metastasis patients. ${ }^{42}$ Higher RANK mRNA levels in primary breast tumors $(n=295)$ were significantly correlated with shorter overall survival. ${ }^{57}$ Similarly, an analysis of primary breast tumors at the time of diagnosis (I-SPY 1 Trial; $n=149$ ) demonstrated that higher RANK expression was not only significantly correlated with poorer recurrence-free survival but was also selectively associated with bone-dominant vs non-bone-dominant recurrence. ${ }^{58}$ Using RT-PCR, analysis of primary renal cell carcinoma (RCC) samples $(n=96)$ indicated that high expression of RANKL or RANK mRNA was correlated with a significantly shorter disease-free survival and shorter bone metastasis survival. ${ }^{59}$ In addition, lower OPG levels were significantly associated with poorer bone metastasis survival.

One study has reported that $89 \%$ of bone metastasis samples (total $n=74$, representing at least 13 different tumor types) expressed RANK protein using immunohistochemistry. ${ }^{60}$ Santini et al. ${ }^{60}$ also indicated that the incidence of RANK protein expression was very high (68\%) in primary tumor samples and the degree/incidence of RANK expression was not significantly different when compared between primary and bone metastatic samples. Although this study reports RANK protein expression in a variety of primary tumors and bone metastases, the extent of expression observed (for example, $65 \%$ for primary breast tumors) is considerably higher than that reported by our own groups $(<20 \%$ for $\mathrm{ER}+/ \mathrm{PR}+$ and $<50 \%$ for ER $-/ \mathrm{PR}-$ primary breast tumors). ${ }^{38,44}$ It will be important to resolve whether these discrepancies are due to differences in the specificity of immunohistochemistry achieved with different reagents/methodologies, independent scoring systems or differences in tumor histotype distributions analyzed in the different studies.

Interestingly, with the exception of the RCC study, ${ }^{59}$ none of the aforementioned studies showed any association of primary tumor RANKL levels, as single variables, with patient outcome. This may reflect relatively low levels and/or infrequent incidence of RANKL expression in primary tumors or limitations in mRNA detection for RANKL using different mRNA detection platforms. The progesterone-dependent regulation of RANKL in breast also indicates that RANKL levels will be dynamically regulated over the menstrual cycle. Alternatively, RANKL levels in the primary tumor may not be an important driver of metastasis, as high baseline RANKL levels are observed in specific tissue sites of distant metastases, such as peripheral lymph nodes, lung and bone. ${ }^{61}$ Activation of the $\beta 2$ adrenergic receptor, common in chronic stress and depression, increases RANKL levels in osteoblasts/bone stroma as well as in the lung and liver tissue. ${ }^{12}$ Altogether, these sources could plausibly provide a local prometastatic signal to RANK-expressing cells. The higher functional expression of RANK in the primary tumor may therefore be a more critical determinant of metastatic functionality conferred by the RANKL/RANK pathway. Although there are reports of soluble RANKL in serum of cancer patients, it is still not clear whether this provides a relevant pathophysiological signal for metastasis. Comparative analysis of circulating vs tissue RANKL has indicated that the systemic and local regulation of RANKL production are discrete processes, ${ }^{62,63}$ suggesting that the local source of RANKL may be more relevant for metastatic disease.

\section{Clinical Bone Metastasis Studies with Denosumab}

Denosumab is a fully human IgG2 monoclonal antibody that binds human RANKL with high affinity. ${ }^{1}$ In an integrated analysis of three randomized double-blind phase 3 trials, denosumab was shown to be superior to the prior standard of care, zoledronic acid, for the prevention or delay of skeletal complications in patients with advanced cancer and bone metastases. ${ }^{64}$ In an additional study in men with nonmetastatic castration-resistant prostate cancer, denosumab prolonged bone metastasis-free survival (hazard ratio $(\mathrm{HR})=0.85$; $95 \%$ confidence interval $(\mathrm{Cl}): 0.73,0.98 ; P=0.028)$ and delayed the time to first bone metastasis $(\mathrm{HR}=0.84 ; 95 \% \mathrm{Cl}: 0.71$, $0.98 ; P=0.032$ ) compared with placebo. ${ }^{65}$ This is the first clinical study successfully demonstrating that targeting the bone compartment makes this microenvironment less hospitable for metastatic establishment and progression. Taken together, these clinical data demonstrate that RANKL inhibition can block osteoclast-mediated bone destruction and 
provides a potential new approach for the treatment of bone metastases.

The abovementioned clinical studies were designed to address skeletal effects and were not designed to test an anticancer effect of denosumab. Interestingly, a post hoc analysis indicated that treatment with denosumab was associated with improved overall survival compared with zoledronic acid in a subgroup of patients with lung cancer from the phase 3 trial of denosumab versus zoledronic acid for the prevention of skeletal-related events (SREs) in patients with bone metastases from advanced solid tumors or multiple myeloma. ${ }^{66,67}$ The significantly improved survival observed among patients with advanced lung cancer who received denosumab could be explained by a greater decrease in bone turnover achieved with denosumab compared with zoledronic acid. In addition, the mechanisms described in this review, such as interruption of tumor cell interactions in the bone microenvironment or direct inhibition of RANKL action on RANKexpressing lung tumor cells, may also have contributed to this finding. ${ }^{67}$

\section{Conclusions and Future Directions}

In summary, preclinical studies have demonstrated the central involvement of RANKL in the establishment and progression of the bone tumor in addition to tumor-induced bone destruction. Clinical studies have extended these observations and proven the causal role of RANKL-mediated osteoclastogenesis in skeletal complications in patients with existing bone metastases as well as the establishment of new bone metastases. In addition to expression within the bone stroma and certain tumor types, RANKL is now known to be expressed within osteocytes or activated Tcells, although the specific contribution of RANKL within these cell types to bone metastasis is yet to be established. Beyond the well-characterized ability to reduce skeletal metastasis, a review of the recent literature provides evidence, with both orthotopic and transgenic mouse spontaneous metastasis models, that RANKL inhibition reduces nonskeletal metastasis.

Future preclinical studies will be important to determine whether any of the individual pro-metastatic mechanisms directly induced by RANKL on RANK-expressing cells in vitro would explain the profound ability of RANKL inhibition to block distant metastasis to nonskeletal sites in vivo. Alternatively, the direct pro-metastatic effect of RANKL may be due to multiple, pleiotropic RANKL-dependent responses acting in concert on RANK-expressing cells. The various effects, including increased migration/invasion, increased EMT induction, increased MMP production, increased levels of angiogenic factors, decreased levels of protease inhibitors, increased survival of a metastatic stem cell and so on would be predicted to enhance metastatic behavior independent of tissue site. Although it is clear that RANK expression on tumor or stem/ progenitor cells is necessary for the pro-metastatic stimuli, the relevant source(s) of RANKL is still under investigation. Acting in an autocrine manner, RANKL may stimulate tumor cell migration and invasion of RANK-expressing cancer cells, as reported for prostate cancer $\mathrm{C} 42 \mathrm{~b}$ cells in vitro for instance. ${ }^{68}$ This autocrine effect may be most relevant in tumor types that frequently express RANK and RANKL, such as lung adenocarcinoma. ${ }^{69}$ Further analysis of RANK and RANKL expression in primary or metastatic sites, using well-validated reagents, may provide additional insights into the relationship of this pathway and metastatic outcomes in patients and molecular mechanisms that influence RANK and RANKL expression.

In breast cancer, preclinical observations suggest that RANKL promotes metastasis via two distinct but overlapping mechanisms: stimulation of the tumor-induced osteoclastogenesis-associated bone metastasis and stimulation of RANKexpressing tumor cells. In addition, the newly defined role for RANKL in normal stem and progenitors cells of the breast, along with the known ability of RANK signaling to regulate canonical and noncanonical NF- $\kappa B$ pathways associated with selfrenewal and activity of CSCs, has prompted the hypothesis that the profound antimetastatic effect of RANKL inhibition observed in the MMTV-neu mammary cancer models in vivo may also include mechanisms acting via decreased activity or survival of the TIC or metastasis-initiating cells.

The efficacy of RANKL inhibition has been clearly demonstrated in the prevention of SREs in patients with bone metastasis caused by a variety of tumors, but given the breastspecific effects of RANKL, breast cancer may provide a unique opportunity to investigate both osteoclast-independent and -dependent pre-metastatic activities of RANKL. Currently, denosumab is being tested to determine whether RANKL inhibition prolongs bone metastasis-free survival and diseasefree survival in the adjuvant breast cancer setting. ${ }^{70}$

\section{Conflict of Interest}

WD is an employee of Amgen Inc. The remaining authors declare no conflict of interest.

\section{Acknowledgements}

We acknowledge Tony Polverino and Dan Branstetter for critical review of the manuscript and Wanda Krall, $\mathrm{PhD}$ for editorial assistance.

\section{References}

1. Lacey DL, Boyle WJ, Simonet WS, Kostenuik PJ, Dougall WC, Sullivan JK et al. Bench to bedside: elucidation of the OPG-RANK-RANKL pathway and the development of denosumab. Nat Rev Drug Discov 2012;11:401-419.

2. Dougall WC, Glaccum M, Charrier K, Rohrbach K, Brasel K, De Smedt T et al. RANK is essential for osteoclast and lymph node development. Genes Dev 1999;13:2412-2424.

3. Kong YY, Yoshida H, Sarosi I, Tan HL, Timms E, Capparelli C et al. OPGL is a key regulator of osteoclastogenesis, lymphocyte development and lymph-node organogenesis. Nature 1999:397:315-323.

4. Lacey DL, Timms E, Tan HL, Kelley MJ, Dunstan CR, Burgess T et al. Osteoprotegerin ligand is a cytokine that regulates osteoclast differentiation and activation. Cell 1998;93:165-176.

5. Simonet WS, Lacey DL, Dunstan CR, Kelley M, Chang MS, Luthy R et al. Osteoprotegerin: a novel secreted protein involved in the regulation of bone density. Cell 1997;89:309-319.

6. Mundy GR. Metastasis to bone: causes, consequences and therapeutic opportunities. Nat Rev Cancer 2002;2:584-593.

7. Weilbaecher KN, Guise TA, McCauley LK. Cancer to bone: a fatal attraction. Nat Rev Cancer 2011:11:411-425

8. Fata JE, Kong YY, Li J, Sasaki T, Irie-Sasaki J, Moorehead RA et al. The osteoclast differentiation factor osteoprotegerin-ligand is essential for mammary gland development. Cell 2000;103:41-50

9. Beleut M, Rajaram RD, Caikovski M, Ayyanan A, Germano D, Choi Y et al. Two distinct mechanisms underlie progesterone-induced proliferation in the mammary gland. Proc Natl Acad Sci USA 2010;107:2989-2994.

10. Tanos T, Sflomos G, Echeverria PC, Ayyanan A, Gutierrez M, Delaloye JF et al. Progesterone/ RANKL is a major regulatory axis in the human breast. Sci Transl Med 2013:5:182ra55.

11. Wood CE, Branstetter D, Jacob AP, Cline JM, Register TC, Rohrbach K et al. Progestin effects on cell proliferation pathways in the postmenopausal mammary gland. Breast Cancer Res 2013;15:R62. 
12. Campbell JP, Karolak MR, Ma Y, Perrien DS, Masood-Campbell SK, Penner NL et al. Stimulation of host bone marrow stromal cells by sympathetic nerves promotes breast cancer bone metastasis in mice. PLOS Biol 2012;10:e1001363.

13. Asselin-Labat ML, VaillantF, Sheridan JM, Pal B, Wu D, Simpson ER et al. Control of mammary stem cell function by steroid hormone signalling. Nature 2010;465:798-802.

14. Joshi PA, Jackson HW, Beristain AG, Di Grappa MA, Mote PA, Clarke CL et al. Progesterone induces adult mammary stem cell expansion. Nature 2010;465:803-807.

15. Nakamura H, Hiraga T, Ninomiya T, Hosoya A, Fujisaki N, Yoneda T et al. Involvement of cellcell and cell-matrix interactions in bone destruction induced by metastatic MDA-MB-231 human breast cancer cells in nude mice. J Bone Miner Metab 2008;26:642-647.

16. Ishikuro M, Sakamoto K, Kayamori K, Akashi T, Kanda H, Izumo T et al. Significance of the fibrous stroma in bone invasion by human gingival squamous cell carcinomas. Bone 2008;43:621-627.

17. Nannuru KC, Futakuchi M, Sadanandam A, Wilson TJ, Varney ML, Myers KJ et al. Enhanced expression and shedding of receptor activator of NF- $\mathrm{KB}$ ligand during tumor-bone interaction potentiates mammary tumor-induced osteolysis. Clin Exp Metastasis 2009;26: 797-808.

18. Thomas RJ, Guise TA, Yin JJ, Elliott J, Horwood NJ, Martin TJ et al. Breast cancer cells interact with osteoblasts to support osteoclast formation. Endocrinology 1999;140: 4451-4458.

19. Dougall WC. Molecular pathways: osteoclast-dependent and osteoclast-independent roles of the RANKL/RANK/OPG pathway in tumorigenesis and metastasis. Clin Cancer Res 2012;18:326-335

20. Sambandam Y, Sundaram K, Liu A, Kirkwood KL, Ries WL, Reddy SV. CXCL13 activation of $c-M y c$ induces RANK ligand expression in stromal/preosteoblast cells in the oral squamous cell carcinoma tumor-bone microenvironment. Oncogene 2013:32:97-105.

21. Bu G, Lu W, Liu CC, Selander K, Yoneda T, Hall C et al. Breast cancer-derived Dickkopf1 inhibits osteoblast differentiation and osteoprotegerin expression: implication for breast cancer osteolytic bone metastases. Int J Cancer 2008:123:1034-1042.

22. Lu X, Wang Q, Hu G, Van Poznak C, Fleisher M, Reiss M et al. ADAMTS1 and MMP1 proteolytically engage EGF-like ligands in an osteolytic signaling cascade for bone metastasis. Genes Dev 2009:23:1882-1894.

23. Rachner TD, Schoppet M, Niebergall U, Hofbauer LC. 17beta-Estradiol inhibits osteoprotegerin production by the estrogen receptor-alpha-positive human breast cancer cell line MCF-7. Biochem Biophys Res Commun 2008;368:736-741.

24. Fradet A, Sorel H, Bouazza L, Goehrig D, Depalle B, Bellahcene A et al. Dual function of ERRalpha in breast cancer and bone metastasis formation: implication of VEGF and osteoprotegerin. Cancer Res 2011;71:5728-5738.

25. Zhang J, Lu Y, Dai J, Yao Z, Kitazawa R, Kitazawa S et al. In vivo real-time imaging of TGF-beta-induced transcriptional activation of the RANK ligand gene promoter in intraosseous prostate cancer. Prostate 2004:59:360-369.

26. Yamada T, Tsuda M, Takahashi T, Totsuka Y, Shindoh M, Ohba Y. RANKL expression specifically observed in vivo promotes epithelial mesenchymal transition and tumor progression. Am J Pathol 2011:178:2845-2856.

27. Zhang X, Junior CR, Liu M, Li F, D'Silva NJ, Kirkwood KL. Oral squamous carcinoma cells secrete RANKL directly supporting osteolytic bone loss. Oral Oncol 2013;49:119-128.

28. Gupta A, Cao W, Chellaiah MA. Integrin alphavbeta3 and CD44 pathways in metastatic prostate cancer cells support osteoclastogenesis via a Runx2/Smad 5/receptor activator of NF- $\mathrm{KB}$ ligand signaling axis. Mol Cancer 2012;11:66.

29. Tang ZN, Zhang F, Tang P, Qi XW, Jiang J. Hypoxia induces RANK and RANKL expression by activating HIF-1alpha in breast cancer cells. Biochem Biophys Res Commun 2011; 408:411-416.

30. van der Pluijm G. Epithelial plasticity, cancer stem cells and bone metastasis formation. Bone 2011:48:37-43.

31. Zhau HE, Odero-Marah V, Lue HW, Nomura T, Wang R, Chu G et al. Epithelial to mesenchymal transition (EMT) in human prostate cancer: lessons learned from ARCaP model. Clin Exp Metastasis 2008;25:601-610.

32. Zhang J, Dai J, Qi Y, Lin DL, Smith P, Strayhorn C et al. Osteoprotegerin inhibits prostate cancer-induced osteoclastogenesis and prevents prostate tumor growth in the bone. $\mathrm{J}$ Clin Invest 2001;107:1235-1244.

33. Morrissey C, Kostenuik PL, Brown LG, Vessella RL, Corey E. Host-derived RANKL is responsible for osteolysis in a C4-2 human prostate cancer xenograft model of experimental bone metastases. BMC Cancer 2007;7:148.

34. Sato K, Lee JW, Sakamoto K, limura T, Kayamori K, Yasuda H et al. RANKL synthesized by both stromal cells and cancer cells plays a crucial role in osteoclastic bone resorption induced by oral cancer. Am J Pathol 2013;182:1890-1899.

35. Roodman GD, Dougall WC. RANK ligand as a therapeutic target for bone metastases and multiple myeloma. Cancer Treat Rev 2008;34:92-101.

36. Brown HK, Ottewell PD, Evans CA, Holen I. Location matters: osteoblast and osteoclast distribution is modified by the presence and proximity to breast cancer cells in vivo. Clin Exp Metastasis 2012;29:927-938.

37. Canon JR, Roudier M, Bryant R, Morony S, Stolina M, Kostenuik PJ et al. Inhibition of RANKL blocks skeletal tumor progression and improves survival in a mouse model of breast cancer bone metastasis. Clin Exp Metastasis 2008;25:119-129.

38. Gonzalez-Suarez E, Jacob AP, Jones J, Miller R, Roudier-Meyer MP, Erwert R et al. RANK ligand mediates progestin-induced mammary epithelial proliferation and carcinogenesis. Nature 2010;468:103-107.
39. Schramek D, Leibbrandt A, Sigl V, Kenner L, Pospisilik JA, Lee HJ et al. Osteoclast differentiation factor RANKL controls development of progestin-driven mammary cancer. Nature 2010:468:98-102.

40. Pellegrini $\mathrm{P}$, Cordero A, Gallego $\mathrm{M}$, Dougal WCl, Munoz $\mathrm{P}$, Pujana $\mathrm{M}$ et al. Constitutive activation of RANK disrupts mammary cell fate leading to tumorigenesis. Stem Cells 2013:31:1954-1965.

41. Jones DH, Nakashima T, Sanchez OH, Kozieradzki I, Komarova SV, Sarosi I et al. Regulation of cancer cell migration and bone metastasis by RANKL. Nature 2006;440:692-696.

42. Martin TJ, Mundy GR. Bone metastasis: can osteoclasts be excluded? Nature 2007;445:E19 discussion E19-20.

43. Tan W, Zhang W, Strasner A, Grivennikov S, Cheng JQ, Hoffman RM et al. Tumour-infiltrating regulatory $T$ cells stimulate mammary cancer metastasis through RANKL-RANK signalling. Nature 2011;470:548-553

44. Palafox M, Ferrer I, Pellegrini P, Vila S, Hernandez-Ortega S, Urruticoechea A et al. RANK induces epithelial-mesenchymal transition and stemness in human mammary epithelial cells and promotes tumorigenesis and metastasis. Cancer Res 2012:72:2879-2888.

45. Blake ML, Tometsko M, Miller R, Jones JC, Dougall WC. RANK expression on breast cancer cells promotes skeletal metastasis. Clin Exp Metastasis 2013;31:233-245.

46. Casimiro S, Mohammad KS, Pires R, Tato-Costa J, Alho I, Teixeira R et al. RANKL/RANK MMP-1 molecular triad contributes to the metastatic phenotype of breast and prostate cancer cells in vitro. PLoS One 2013:8:e63153.

47. Mori K, Le Goff B, Charrier C, Battaglia S, Heymann D, Redini F. DU145 human prostate cancer cells express functional receptor activator of $\mathrm{NF} K \mathrm{~B}$ : new insights in the prostate cancer bone metastasis process. Bone 2007:40:981-990.

48. Armstrong AP, Miller RE, Jones JC, Zhang J, Keller ET, Dougall WC. RANKL acts directly on RANK-expressing prostate tumor cells and mediates migration and expression of tumor metastasis genes. Prostate 2008;68:92-104.

49. Zhang L, Teng Y, Zhang Y, Liu J, Xu L, Qu J et al. C-Src-mediated RANKL-induced breast cancer cell migration by activation of the ERK and Akt pathway. Oncol Lett 2012; 3:395-400.

50. Chen LM, Kuo CH, Lai TY, Lin YM, Su CC, Hsu HH et al. RANKL increases migration of human lung cancer cells through intercellular adhesion molecule-1 up-regulation. J Cell Biochem 2011;112:933-941.

51. Rucci N, Millimaggi D, Mari M, Del Fattore A, Bologna M, Teti A et al. Receptor activator of NF- $\mathrm{KB}$ ligand enhances breast cancer-induced osteolytic lesions through upregulation of extracellular matrix metalloproteinase inducer/CD147. Cancer Res 2010;70 $6150-6160$.

52. Luo JL, Tan W, Ricono JM, Korchynskyi O, Zhang M, Gonias SL et al. Nuclear cytokineactivated IKKalpha controls prostate cancer metastasis by repressing Maspin. Nature 2007;446:690-694.

53. Cao Y, Luo JL, Karin M. IKB kinase alpha kinase activity is required for self-renewal of ErbB2/Her2-transformed mammary tumor-initiating cells. Proc Natl Acad Sci USA 2007;104:15852-15857.

54. Kendellen MF, Bradford JW, Lawrence CL, Clark KS, Baldwin AS. Canonical and noncanonical NF- $\mathrm{KB}$ signaling promotes breast cancer tumor-initiating cells. Oncogene 2013;33:1297-1305.

55. Chu JE, Allan AL. The role of cancer stem cells in the organ tropism of breast cancer metastasis: a mechanistic balance between the 'seed' and the 'soil'? Int J Breast Cancer 2012;2012:209748.

56. Ithimakin S, Day KC, Malik F, Zen Q, Dawsey SJ, Bersano-Begey TF et al. HER2 drives luminal breast cancer stem cells in the absence of HER2 amplification: implications for efficacy of adjuvant trastuzumab. Cancer Res 2013;73:1635-1646.

57. Santini D, Schiavon G, Vincenzi B, Gaeta L, Pantano F, Russo A et al. Receptor activator of $\mathrm{NF}-\mathrm{KB}$ (RANK) expression in primary tumors associates with bone metastasis occurrence in breast cancer patients. PLoS One 2011;6:e19234.

58. LiJ, Yau C, Campbell M, Esserman L, Park JW, Rugo HS et al. Correlation of receptor activator of nuclear factor kappa $b$ (RANK) expression in breast cancer $(\mathrm{BC})$ at the time of diagnosis with recurrence-free survival (RFS) and risk of bone-dominant metastases (BDM) in the ISPY1 trial. J Clin Oncol2012;30(Suppl 27):abstr. 2. (Available at: http://meeting.ascopubs.org/cgi/content/ abstract/30/27_suppl/2?sid=007510f1-1d3c-4894-89ca-8a271491d3de).

59. Mikami S, Katsube K, Oya M, Ishida M, Kosaka T, Mizuno R et al. Increased RANKL expression is related to tumour migration and metastasis of renal cell carcinomas. J Pathol 2009;218: 530-539.

60. Santini D, Perrone G, Roato I, Godio L, Pantano F, Grasso D et al. Expression pattern of receptor activator of $N F_{K} B(R A N K)$ in a series of primary solid tumors and related bone metastases. J Cell Physiol 2011;226:780-784

61. Kartsogiannis V, Zhou H, Horwood NJ, Thomas RJ, Hards DK, Quinn JM et al. Localization of RANKL (receptor activator of NF kappa B ligand) mRNA and protein in skeletal and extraskeletal tissues. Bone 1999:25:525-534.

62. Stolina M, Bolon B, Middleton S, Dwyer D, Brown H, Duryea D et al. The evolving systemic and local biomarker milieu at different stages of disease progression in rat adjuvant-induced arthritis. J Clin Immunol 2009:29:158-174.

63. Findlay DM, Atkins GJ. Relationship between serum RANKL and RANKL in bone. Osteoporos Int 2011;22:2597-2602.

64. Lipton A, Fizazi K, Stopeck AT, Henry DH, Brown JE, Yardley DA et al. Superiority of denosumab to zoledronic acid for prevention of skeletal-related events: a combined analysis of 3 pivotal, randomised, phase 3 trials. Eur J Cancer 2012;48:3082-3092. 
65. Smith MR, Saad F, Coleman R, Shore N, Fizazi K, Tombal B et al. Denosumab and bonemetastasis-free survival in men with castration-resistant prostate cancer: results of a phase 3 , randomised, placebo-controlled trial. Lancet 2012;379:39-46.

66. Henry DH, Costa L, Goldwasser F, Hirsh V, Hungria V, Prausova J et al. Randomized, doubleblind study of denosumab versus zoledronic acid in the treatment of bone metastases in patients with advanced cancer (excluding breast and prostate cancer) or multiple myeloma. J Clin Oncol 2011;29:1125-1132.

67. Scagliotti GV, Hirsh V, Siena S, Henry DH, Woll PJ, Manegold C et al. Overall survival improvement in patients with lung cancer and bone metastases treated with denosumab versus zoledronic acid: subgroup analysis from a randomized phase 3 study. $J$ Thorac Oncol 2012;7:1823-1829.

68. Sabbota AL, Kim HR, Zhe X, Fridman R, Bonfil RD, Cher ML. Shedding of RANKL by tumorassociated MT1-MMP activates Src-dependent prostate cancer cell migration. Cancer Res 2010;70:5558-5566
69. Branstetter D, Huang L-Y, Dougal WCI. RANK and RANK-Ligand (RANKL) expression in primary human lung cancer. J Thorac Oncol 2013;8:S446.

70. Goss PE, Barrios CH, Chan A, Finkelstein DM, Iwata H, Martin M et al. Denosumab versus placebo as adjuvant treatment for women with early-stage breast cancer at high risk of disease recurrence (D-CARE): an international, randomized, double-blind, placebo-controlled phase 3 clinical trial. Cancer Res 2012;72(24 Suppl):Abstract number OT2-3-03. (Available at: http://cancerres.aacrjournals.org/cgi/content/meeting_abstract/72/24_MeetingAbstracts/OT23-03?sid=f0763b5d-469a-48da-ae0e-a4c15e0ace37).

cC) (1) $\Theta$ This work is licensed under a Creative Commons AttributionConCommercial-NoDerivs 3.0 Unported License. To view a copy of this license, visit http://creativecommons.org/licenses/by-nc-nd/3.0/ 